\title{
Comparison of High Intensity Accelerated Corneal Cross Linking Protocols in Treatment of Progressive Keratoconus
}

\author{
Bushra Akbar', Imran Basit ${ }^{2}$, Amjad Akram ${ }^{3}$, Maham Zahid ${ }^{4}$ \\ ${ }^{1,2}$ Armed Forces Institute of Ophthalmology, Rawalpindi. ${ }^{3}$ Combined Military Hospital (CMH), Kharian \\ ${ }^{4}$ National University of Medical Sciences (NUMS), Rawalpindi - Pakistan
}

\begin{abstract}
Purpose: To compare the safety and efficacy of Accelerated Corneal Cross Linking (AXL) protocols, $9 \mathrm{~mW} / \mathrm{cm}^{2}$ for $10 \mathrm{~min}$ with $18 \mathrm{~mW} / \mathrm{cm}^{2}$ for $5 \mathrm{~min}$ in terms of refractive and topographic keratometric indices in patients with progressive Keratoconus.
\end{abstract}

Study Design: Quasi experimental study.

Place and Duration of Study: Armed Forces Institute of Ophthalmology, Rawalpindi Pakistan, from Nov 2016 to Jun 2018.

Methods: Sixty eyes, 30 in each group, of 55 consecutive patients diagnosed with progressive keratoconus were enrolled through convenience sampling and were subjected to AXL with irradiance protocols of $18 \mathrm{mWatt} / \mathrm{cm}^{2}$ for 5 minutes in group 1 and $9 \mathrm{~mW} / \mathrm{cm}^{2}$ for 10 minutes in group 2. All patients underwent a comprehensive ophthalmic examination at baseline and postoperative follow up visits at 3, 6, 12 and 18 months. Primary outcome parameter was disease stability defined as increase in maximum keratometry over baseline $\mathrm{K}$ max of no more than 1 diopter at 1 year after AXL. Statistical analysis of data was performed with IBM SPSS software (version 20.0 SPSS). $P$ value of $<0.05$ was considered as statistically significant.

Results: Disease stability was $96 \%$ in each group. At the final time point of 18 months, group 2 (AXL $9 \mathrm{~mm}$ watt $/ \mathrm{cm}^{2}$ for $10 \mathrm{~min}$ ) was superior as compared to group 1 (AXL $18 \mathrm{~mm}$ watt $/ \mathrm{cm}^{2}$ for $5 \mathrm{~min}$ ) in terms of flattening of steep and $\operatorname{sim} \mathrm{K}(p=0.007,0.023$ respectively).

Conclusion: The two AXL protocols are safe and appear to show comparable efficacy in disease stability. They can be used alternatively in the treatment of progressive keratoconus.

Key Words: Corneal Cross Linking, Keratoconus, Ultraviolet A, Accelerated Corneal Cross Linking.

How to Cite this Article: Akbar B, Basit I, Akram A, Zahid M. Comparison of High Intensity Accelerated Corneal Cross Linking Protocols in Treatment of Progressive Keratoconus. Pak J Ophthalmol. 2020, 36 (2): 96-102.

Doi: $10.36351 /$ pjo.v36i2.976

\section{INTRODUCTION}

High intensity Accelerated corneal collagen cross

Correspondence: Bushra Akbar

Armed Forces Institute of Ophthalmology, RawalpindiEmail:dr.bushra.akbar@gmail.com

Received: January 2, 2020

Accepted: March 5, 2020 linking protocols (AXL) with optimized beam profiles have been new options in treatment armamentarium of progressive keratoconus. AXL protocols are in accordance with Bunsen-roscoe law of chemical reciprocity that states "same photochemical effect can be achieved by reducing the irradiation interval, provided that the total energy level is kept constant by a corresponding increase in irradiation intensity." 
The classical CXL Dresden protocol included debridement of the corneal epithelium in the central 7 to $9 \mathrm{~mm}$ area and a pre-soak of 30-minute, with riboflavin-5-phosphate and $20 \%$ dextran. It was followed by UVA irradiance of $3 \mathrm{~mW} / \mathrm{cm} 2(365 \mathrm{~nm}$, total energy $5.4 \mathrm{j} / \mathrm{cm}^{2}$ ) for 30 minutes. ${ }^{2}$ AXL protocols incorporated a fraction of time of Dresden protocol, with a proportionate increase in irradiation intensity in order to achieve total energy levels of $5.4 \mathrm{j} / \mathrm{cm}^{2}$ equivalent to that proposed by Dresden classical protocol i.e. $9 \mathrm{~mW} / \mathrm{cm}^{2}$ for 10 minutes, $18 \mathrm{~m} \mathrm{~W} / \mathrm{cm}^{2}$ for 5 minutes and $30 \mathrm{~m} \mathrm{~W} / \mathrm{cm}^{2}$ for 3 minutes. ${ }^{3}$ The reduced surgical time of AXL confers the benefit of increased patient and surgeon comfort, minimal corneal dehydration and a decreased risk of postoperative infection owing to less exposure of denuded corneal epithelium. ${ }^{4}$ The same photochemical effect or photoactivated corneal covalent intra and interlamellar stromal cross linking achieved by AXL and conventional protocols promise comparable efficacy in terms of disease stabilization and a relative improvement in topographic keratometric indices and refractive profiles by augmenting the biomechanical strength of cornea. ${ }^{3-5}$

Previous comparative clinical trials conducted with variable beam profiles, with different riboflavin solutions, in different populations have claimed safety and comparable efficacy of conventional protocol vs. AXL algorithms. ${ }^{5-9} \mathrm{We}$ aimed to directly compare the safety and efficacy of AXL protocols, $9 \mathrm{mw} / \mathrm{cm}^{2}$ for 10 minutes with $18 \mathrm{~mW} / \mathrm{cm}^{2}$ for 5 minutes in terms of refractive and topographic keratometric indices, in patients with progressive keratoconus.

\section{METHODS}

This study was conducted at Armed Forces Institute of Ophthalmology, Rawalpindi Pakistan, from Nov 2016 to Jun 2018 after approval from Hospital ethical committee and, in accordance with tenets of declaration of Helsinki. Sixty eyes of 55 consecutive patients diagnosed with progressive keratoconus were enrolled in this study after obtaining an informed written consent. Thirty eyes, each were randomized to AXL with irradiance protocols of $18 \mathrm{mWatt} / \mathrm{cm}^{2}$ for 5 minutes in group 1 and $9 \mathrm{mWatt} / \mathrm{cm}^{2}$ for 10 minutes in group 2. Inclusion criteria were; clear cornea, age between 18 to 40 years, documented evidence or reported progression with reduced visual acuity by more than 0.50 Snellen lines, an increase in the spherical/cylinder refraction of more than $0.50 \mathrm{D}$, an increase in the maximum keratometry reading of more than $1 \mathrm{D}$ and a reduction in CCT of more than $10 \mu \mathrm{m}$ over the previous 12 months; documented on a minimum of 2 corneal topographies over a period of 6 months. Any active or previous ocular infections, corneal opacities, dry eyes, corneal pachymetry of less than 400 microns at thinnest point, previous CXL treatment or any ocular surgery, active autoimmune disorders, pregnancy and lactation were excluded from the study.

All patients underwent a comprehensive ophthalmic examination at baseline and postoperative follow up visits at 3, 6, 12 and 18 months, which included UDVA (uncorrected distance visual acuity), CDVA (corrected distance visual acuity) (Snellen visual acuity converted to $\log$ MAR notation), slit lamp biomicroscopy, dual scheimpflug corneal topography (Galilie G4), pachymetry (Galilie G4), specular microscopy (Topcon sp-3000, USA) for endothelial cell density analysis and dilated fundus examination. Rigid gas permeable contact lenses were discontinued for three weeks and soft contact lens for at least two weeks prior to baseline evaluation.

AXL was performed under topical anaesthesia $0.05 \%$ proparacaine hydrochloride (Alkaine), in both groups as a day care procedure. Standard preoperative preparation with $5 \%$ povidine iodine solution was done. Central $9 \mathrm{~mm}$ of epithelium was scraped off, followed by instillation of one drop of isotonic riboflavin ( $0.1 \%$ riboflavin (Vit B2), HPMC $1.1 \%$ (Peschke M, PESCHKE Trade GmbH) every 2 minutes for 20 minutes. Cornea with pachymetry of less than 400 microns after epithelium removal and isotonic riboflavin instillation were treated with hypotonic riboflavin drops Peschke $\mathrm{H}$ (PESCHKE Trade $\mathrm{GmbH}$ ) one drop every 5 seconds till it reached 400 microns. Cornea was exposed to UVA light of 366-370 microns at a distance of $55 \mathrm{~mm}$ from the eye at an irradiance of $18 \mathrm{~mW} / \mathrm{cm}^{2}$ for 5 minutes in group 1 and $9 \mathrm{mWatt} / \mathrm{cm}^{2}$ for 10 minutes in group 2, delivering a total energy of 5.4 joules/cm ${ }^{2}$ (CCL VARIO 365, PESCHKE Trade GmbH, Huenenberg Switzerland) in both groups, with continued instillation of riboflavin drops every 2 minutes. A bandage contact lens (Interojo, Korea) was applied and removed on $7^{\text {th }}$ postoperative day, if epithelium had healed.

Post operatively cyclopentolate $1 \%$ eye drops were advised 8 hourly for 3 days and moxifloxacin (Vigamox $0.05 \%$, Alcon) for 2 weeks respectively. Topical steroids Flourometholone (FML 0.1\%, 
Allergen) were added after one week, if epithelium had healed and continued for 4 weeks along with topical lubricants hypromellose, dextran (Tear Natural II, Alcon).

Primary outcome parameter was disease stability defined as increase in maximum keratometry over baseline Kmax of no more than 1 diopter at 1 year after AXL. Refractive outcome UDVA, CDVA, spherical equivalent SE, Refractive astigmatism, change in simulated $K$ and steep $K$, corneal pachymetry (central corneal thickness) were additional outcome measures documented at 3, 6, 12 and 18 months post AXL test points.

Statistical analysis of data was performed with IBM SPSS software (version 20.0 SPSS). $p$ value of $<0.05$ was considered as statistically significant. Normality of data was established utilizing Kolmogorov smirnov test. Within the groups, related two samples comparisons at multiple points were performed with one way repeated measure ANOVA for normally distributing data and Wilcoxon matched pairs test for non-normally distributing data. Between the group comparisons for normally distributing data was done with independent sample t test and Mann Whitney $U$ test was applied to non-normal distributions.

\section{RESULTS}

The mean age was $24.47 \pm 4.90$ years in AXL group 1 and 24.81 \pm 6.39 in AXL group 2, with no statistically significant difference. Keratometric parameters (simulated K, steep K, Kmax), refractive data (UDVA, CDVA, refractive astigmatism, spherical equivalent SE) and pachymetry, central corneal thickness (CCT) were comparable at baseline between the two AXL groups (Table 1). The mean postoperative log MAR UDVA improved at test points of 6,12 and 18 months in both AXL groups, however these differences were not statistically significant $(p=0.361$ and $p=0.138$ respectively) (Table 2 and 3). The mean postoperative $\log$ MAR CDVA significantly improved over mean preoperative value in both the groups $(p=0.020$ and $p=0.020$ ). However, the difference in post-operative $\log$ MAR UDVA and CDVA was not statistically significant between the AXL groups at any time point $(p=0.979, \quad 0.700,0.873,0.125,0.072, \quad 0.171$ respectively) (Table 4). The refractive Astigmatism and spherical equivalent SE showed significant reduction from baseline at all postoperative test points of 3, 6, 12 and 18 months in both groups $(p<0.05)$. Astigmatism reached significance in group 2 only at

Table 1: Preoperative Comparison of Parameters.

\begin{tabular}{lccc}
\hline \multirow{2}{*}{ Parameters } & \multicolumn{2}{c}{ Accelerated CXL } & P \\
& GP 1 $(\mathbf{n = 3 0})$ & GP 2 $(\mathbf{n = 3 0})$ & value \\
\hline UCVA & $0.75 \pm 0.51$ & $0.65 \pm 0.58$ & 0.486 \\
CDVA & $0.37 \pm 0.27$ & $0.26 \pm 0.22$ & 0.090 \\
SE & -6.05 V 3.08 & $-6.2 \pm 2.85$ & 0.781 \\
Ast & $-4.15 \pm 1.69$ & $-3.81 \pm 5.52$ & 0.540 \\
Steep K & $50.4 \pm 3.72$ & $49.6 \pm 3.53$ & 0.404 \\
Sim K & $48.37 \pm 2.96$ & $47.8 \pm 2.92$ & $0.379^{\mathrm{b}}$ \\
K $_{\text {max }}$ & $53.97 \pm 4.29$ & $53.63 \pm 4.80$ & 0.774 \\
CCT & $471.20 \pm 36.91$ & $479 \pm 37.7$ & $0.442^{\mathrm{b}}$ \\
ECD & $2566.19 \pm 428.61$ & $2516.88 \pm 343.85$ & 0.781 \\
\hline
\end{tabular}

${ }^{\mathrm{a}}$ Independent samples T-test, ${ }^{\mathrm{b}}$ Mann-Whitney $\mathrm{U}$ test $* \mathrm{P}<0.05$. UCVA = Uncorrected distance Visual acuity, CDVA = corrected distance visual acuity, $\mathrm{SE}=$ Spherical equivalent, $\mathrm{Ast}=$ Refractive astigmatism, Steep K = Steep keratometry, Sim K = Simulated keratometry, $\mathrm{Kmax}=$ Maximum or Apex Keratometry, $\mathrm{CCT}=$ central corneal thickness

12 and 18 months follow-up ( $p=0.000,0.000)$ (Tables 2, 3). The change in SE and Astigmatism did not differ between two groups (Table 4).

Disease stability that was the primary outcome measure was $96 \%$ in each group in present study. Similar trend of significant flattening of Kmax, sim K, steep K was observed in each group $(p<0.05)$ against baseline indices. At 6 and 12 months follow up, no significant differences in keratometric parameters Kmax, sim K, and steep K were ascertained comparing both procedures. At the final time point of 18 months, group 2 (AXL $9 \mathrm{mWatt} / \mathrm{cm}^{2}$ for 10 minutes) was superior as compared to group 1 (AXL $18 \mathrm{mWatt} / \mathrm{cm}^{2}$ for 5 minutes) in terms of flattening of steep and sim $\mathrm{K}$ ( $p=0.007,0.023$ respectively) and Kmax, that barely missed statistical significance (Table 4). CCT in group 2 showed reduction of $23.16 \pm 21.77$ microns in group 1 and $25.06 \pm 28.18$ in group 2 with no statistically significant difference at any follow-up between the groups ( $p=0.855,0.351,0.771$ respectively) (Table 4).

Intra-group analysis showed significant reduction in endothelial cell count at the end of six, twelve and eighteen months in all groups ( $p<0.05$ in all groups). However, between the groups, the reduction in endothelial cells was not statistically significant at any time point.

In group 2, one eye had a mild stromal haze that resolved with corticosteroid treatment by 6 weeks. No incidence of corneal endothelial de-compensation or any other adverse effect was recorded in any treatment 
Table 2: Comparison of Pre and Post-operative refractive and topographical measurements in AXL Group 1 - (18 $\mathrm{mWatt} / \mathrm{cm}^{2}$ for $5 \mathrm{~min}$ ).

\begin{tabular}{|c|c|c|c|c|c|c|}
\hline \multirow[b]{2}{*}{ Parameters } & \multicolumn{5}{|c|}{ Postoperative Follow-up Time Period } & \multirow[b]{2}{*}{$P$ value } \\
\hline & $\begin{array}{c}\text { Pre-operative } \\
\quad(\mathbf{n}=\mathbf{3 0})\end{array}$ & $\begin{array}{c}3 \text { Months } \\
(\mathbf{n}=\mathbf{3 0})\end{array}$ & $\begin{array}{c}6 \text { Months } \\
(\mathbf{n}=30)\end{array}$ & $\begin{array}{c}12 \text { Months } \\
(\mathrm{n}=\mathbf{3 0})\end{array}$ & $\begin{array}{l}18 \text { Months } \\
(\mathrm{n}=30)\end{array}$ & \\
\hline UCVA & $0.75 \pm 0.51$ & $0.71 \pm 0.47$ & $0.73 \pm 0.46$ & $0.67 \pm 0.45$ & $0.65 \pm 0.43$ & 0.361 \\
\hline CDVA & $0.37 \pm 0.27$ & $0.34 \pm 0.20$ & $0.38 \pm 0.25$ & $0.32 \pm 0.27$ & $0.31 \pm 0.27$ & $0.020 *$ \\
\hline SE & $-6.05 \pm 3.08$ & $-5.05 \pm 2.67$ & $-4.12 \pm 2.57$ & $-4.66 \pm 3.03$ & $-4.60 \pm 2.96$ & $0.000 *$ \\
\hline Ast & $-4.15 \pm 1.69$ & $-3.79 \pm 1.61$ & $-3.48 \pm 1.11$ & $-3.03 \pm 1.20$ & $-2.97 \pm 1.18$ & $0.000 *$ \\
\hline Steep K & $50.48 \pm 3.72$ & $49.95 \pm 3.76$ & $49.71 \pm 3.67$ & $49.31 \pm 3.62$ & $49.18 \pm 3.59$ & $0.000^{*}$ \\
\hline $\operatorname{Sim} K$ & $48.37 \pm 2.96$ & $48.02 \pm 3.20$ & $48.09 \pm 3.18$ & $47.64 \pm 3.18$ & $47.57 \pm 3.20$ & $0.001 *$ \\
\hline $\mathrm{K}_{\max }$ & $53.97 \pm 4.29$ & $53.45 \pm 4.09$ & $53.26 \pm 3.74$ & $52.22 \pm 3.92$ & $52.27 \pm 3.94$ & $0.000 *$ \\
\hline $\mathrm{CCT}$ & $471.20 \pm 36.91$ & $452.60 \pm 43.57$ & $456.96 \pm 44.38$ & $447.40 \pm 43.24$ & $446.50 \pm 44.25$ & $0.000^{*}$ \\
\hline
\end{tabular}

One-way ANOVA repeated measures test, $* \mathrm{P}<0.05 . \mathrm{AXL}=$ Accelerated CXL, UCVA $=$ Visual acuity, CDVA $=$ Corrected distance visual acuity, $\mathrm{SE}=$ Spherical equivalent, Ast $=$ Refractive astigmatism, Steep K = Steep keratometry, Sim K = Simulated keratometry, Kmax $=$ Maximum or Apex Keratometry, CCT=central corneal thickness

Table 3: Comparison of Pre and Post-operative refractive and topographical measurements in AXL Group 2 - $\left(9 \mathrm{mwatt} / \mathrm{cm}^{2}\right.$ for $10 \mathrm{~min}$ ).

\begin{tabular}{|c|c|c|c|c|c|c|}
\hline \multirow[b]{2}{*}{ Parameters } & \multicolumn{5}{|c|}{ Postoperative Follow-up Time Period } & \multirow[b]{2}{*}{ P value } \\
\hline & $\begin{array}{c}\text { Preoperative } \\
(\mathbf{n}=\mathbf{3 0})\end{array}$ & $\begin{array}{c}3 \text { Months } \\
(\mathbf{n}=\mathbf{3 0})\end{array}$ & $\begin{array}{c}6 \text { Months } \\
(\mathrm{n}=\mathbf{3 0}) \\
\end{array}$ & $\begin{array}{c}1 \text { Year } \\
(n=30)\end{array}$ & $\begin{array}{c}18 \text { Months } \\
(\mathrm{n}=30)\end{array}$ & \\
\hline UCVA & $0.65 \pm 0.58$ & $0.73 \pm 0.49$ & $0.62 \pm 0.53$ & $0.60 \pm 0.51$ & $0.56 \pm 0.45$ & 0.138 \\
\hline CDVA & $0.26 \pm 0.22$ & $0.27 \pm 0.20$ & $0.32 \pm 0.25$ & $0.28 \pm 0.27$ & $0.26 \pm 0.25$ & $0.022 *$ \\
\hline SE & $-6.27 \pm 2.85$ & $-5.68 \pm 2.80$ & $-4.52 \pm 2.18$ & $-4.96 \pm 2.84$ & $-4.27 \pm 2.25$ & $0.000 *$ \\
\hline Ast & $-3.81 \pm 2.52$ & $-3.98 \pm 1.87$ & $-3.68 \pm 1.49$ & $-2.62 \pm 2.32$ & $-2.50 \pm 1.26$ & $0.000 *$ \\
\hline Steep K & $49.69 \pm 3.53$ & $49.17 \pm 3.44$ & $48.78 \pm 3.49$ & $48.40 \pm 3.47$ & $47.56 \pm 3.41$ & $0.000 *$ \\
\hline Sim K & $47.84 \pm 2.92$ & $47.36 \pm 2.94$ & $47.10 \pm 3.09$ & $46.72 \pm 2.95$ & $46.07 \pm 3.00$ & $0.000^{*}$ \\
\hline $\mathrm{K}_{\max }$ & $53.63 \pm 4.80$ & $52.95 \pm 4.37$ & $52.94 \pm 4.39$ & $51.70 \pm 4.38$ & $50.92 \pm 4.33$ & $0.001 *$ \\
\hline $\mathrm{CCT}$ & $479.80 \pm 37.75$ & $455.10 \pm 44.72$ & $467.26 \pm 45.97$ & $461.93 \pm 47.99$ & $454.73 \pm 47.05$ & $0.000^{*}$ \\
\hline
\end{tabular}

ANOVA repeated measures test, $* \mathrm{P}<0.05 . \mathrm{AXL}=$ Accelerated $\mathrm{CXL}, \mathrm{UCVA}=$ Uncorrected distance Visual acuity, $\mathrm{CDVA}=\mathrm{Corrected}$ distance visual acuity, $\mathrm{SE}=$ Spherical equivalent, Ast $=$ Refractive astigmatism, Steep K = Steep keratometry, Sim $\mathrm{K}=$ Simulated keratometry, Kmax = Maximum or Apex Keratometry, CCT =central corneal thickness

Table 4: Comparison of Post-operative changes in refractive data, keratometry and pachymetry between two study groups.

\begin{tabular}{|c|c|c|c|c|c|c|c|c|c|}
\hline Parameters & $\begin{array}{c}6 \text { Months } \\
(n=30)\end{array}$ & $\begin{array}{l}12 \text { Months } \\
(\mathrm{n}=30)\end{array}$ & $\begin{array}{l}18 \text { Months } \\
(\mathrm{n}=\mathbf{3 0})\end{array}$ & $\begin{array}{l}6 \text { Months } \\
(\mathrm{n}=30)\end{array}$ & $\begin{array}{l}12 \text { Months } \\
(\mathrm{n}=30)\end{array}$ & $\begin{array}{l}18 \text { Months } \\
(\mathbf{n}=\mathbf{3 0})\end{array}$ & 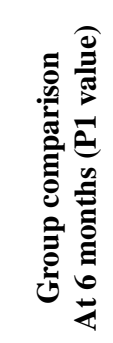 & 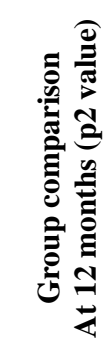 & 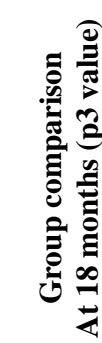 \\
\hline$\overline{\mathrm{UCVA}}$ & $-0.29 \pm 0.27$ & $-0.82 \pm 0.29$ & $-0.82 \pm$ & -0.31 & -0.05 & $\overline{-0.94}$ & 0.979 & 0.700 & 0.873 \\
\hline CDVA & $0.11 \pm 0.15$ & $-0.045 \pm 1.56$ & $-0.45 \pm 0.15$ & & & $0.01 \pm 0.11$ & 0.125 & 0.072 & 0.171 \\
\hline SE & $1.93 \pm 3.29$ & $1.39 \pm 1.04$ & $-5.42 \pm 3.46$ & $1.74 \pm 2.45$ & $1.30 \pm 1.54$ & $-4.93 \pm 2.69$ & 0.803 & 0.793 & 0.548 \\
\hline Ast & $0.67 \pm 1.02$ & $1.12 \pm 0.99$ & $1.12 \pm 0.99$ & $0.12 \pm 1.98$ & $1.18 \pm 3.25$ & $1.31 \pm 2.10$ & 0.189 & 0.922 & 0.660 \\
\hline Steep K & $-0.77 \pm 1.10$ & $-0.77 \pm 1.10$ & $-1.16 \pm 1.40$ & $-0.91 \pm 1.68$ & $-0.91 \pm 1.68$ & $-2.13 \pm 1.77$ & 0.694 & 0.694 & $0.023 *$ \\
\hline Sim K & $-0.28 \pm 1.15$ & $-0.73 \pm 1.44$ & $-0.73 \pm 1.44$ & $-0.73 \pm 1.13$ & $1.11 \pm 1.30$ & $-1.77 \pm 1.42$ & 0.130 & 0.283 & $0.007 *$ \\
\hline $\mathrm{K}_{\max }$ & $-0.70 \pm 2.04$ & $-1.74 \pm 2.34$ & $-1.70 \pm 2.48$ & $-0.68 \pm 1.32$ & $-1.93 \pm 1.51$ & $-2.71 \pm 1.43$ & 0.967 & 0.723 & 0.058 \\
\hline $\mathrm{CCT}$ & $-14.23 \pm 35.45$ & $-23.80 \pm 21.99$ & $-23.16 \pm 21.77$ & $-12.53 \pm 36.27$ & $-17.86 \pm 26.62$ & $-25.06 \pm 28.18$ & 0.855 & 0.351 & 0.771 \\
\hline ECD & $-3.66 \pm 6.00$ & $-4.16 \pm 6.28$ & $-4.16 \pm 6.28$ & $-3.52 \pm 5.99$ & $-4.01 \pm 6.30$ & $-3.44 \pm 6.58$ & 0.926 & 0.929 & 0.670 \\
\hline
\end{tabular}

Independent samples $\mathrm{T}$ test. $* \mathrm{P}<0.05$. AXL $=$ Accelerated CXL, UCVA $=$ Visual acuity, CDVA $=$ Corrected distance visual acuity, $\mathrm{SE}=$ Spherical equivalent, Ast $=$ Refractive astigmatism, Steep K $=$ Steep keratometry, Sim K $=$ Simulated keratometry, Kmax $=$ Maximum or Apex Keratometry, CCT $=$ central corneal thickness, ECD = Endothelial cell count 
Table 5: Previous Clinical Trials of AXL.

\begin{tabular}{|c|c|c|c|c|c|c|c|}
\hline \multirow{2}{*}{ Study } & \multirow{2}{*}{ Study Design } & \multicolumn{2}{|c|}{$\begin{array}{l}\text { Conventional } \\
\text { Cross Linking }\end{array}$} & \multicolumn{2}{|r|}{ Accelerated Cross Linking } & \multirow{2}{*}{$\begin{array}{l}\text { Follow-up } \\
\text { (Months) }\end{array}$} & \multirow{2}{*}{ Findings } \\
\hline & & $\mathbf{N}$ & $\begin{array}{l}\text { Protocol/ } \\
\text { Platform } \\
\end{array}$ & $\mathbf{N}$ & Protocol/Platform & & \\
\hline $\begin{array}{l}\text { Cinar et al. } \\
2014^{7}\end{array}$ & $\begin{array}{l}\text { Prospective } \\
\text { comparative case } \\
\text { series }\end{array}$ & 13 & $\begin{array}{l}3 \mathrm{~mW} / \mathrm{cm}^{2} \\
\text { for } 30 \mathrm{mins}\end{array}$ & 13 & $9 \mathrm{~mW} / \mathrm{cm}^{2}$ for $10 \mathrm{mins}$ & 6 & $\begin{array}{l}\text { Comparable visual and refractive } \\
\text { results, decrease in } \mathrm{Km} \text { and } \\
\text { Kmax in both groups }\end{array}$ \\
\hline $\begin{array}{l}\text { Hashemi } \\
\text { et al. } 2015^{8}\end{array}$ & $\begin{array}{l}\text { Prospective } \\
\text { randomized } \\
\text { comparative case } \\
\text { series }\end{array}$ & 31 & $\begin{array}{l}3 \mathrm{~mW} / \mathrm{cm}^{2} \\
\text { for } 30 \text { mins } \\
U V-X\end{array}$ & 31 & $\begin{array}{l}18 \mathrm{~mW} / \mathrm{cm}^{2} \text { for } 5 \mathrm{mins} \\
\mathrm{UV}-\mathrm{X}\end{array}$ & 6 & $\begin{array}{l}\text { Comparable visual acuity, } \\
\text { refractive, keratometric and } \\
\text { biomechanical outcomes }\end{array}$ \\
\hline $\begin{array}{l}\text { Hashemi } \\
\text { et al. } 2015^{9}\end{array}$ & $\begin{array}{l}\text { Prospective } \\
\text { randomized }\end{array}$ & 31 & $\begin{array}{l}18 \mathrm{~mW} / \mathrm{cm}^{2} \\
\text { for } 5 \mathrm{mins} \\
\mathrm{UV}-\mathrm{X}\end{array}$ & 31 & $\begin{array}{l}3 \mathrm{~mW} / \mathrm{cm}^{2} \text { for } 30 \mathrm{mins} \\
\mathrm{UV}-\mathrm{X}\end{array}$ & 18 & $\begin{array}{l}\text { Comparable visual acuity, } \\
\text { refractive, keratometric and } \\
\text { biomechanical outcomes }\end{array}$ \\
\hline $\begin{array}{l}\text { Chow et al. } \\
2015^{10}\end{array}$ & $\begin{array}{l}\text { Prospective } \\
\text { comparative case } \\
\text { series }\end{array}$ & 19 & $\begin{array}{l}3 \mathrm{~mW} / \mathrm{cm}^{2} \\
\text { for } 30 \mathrm{mins} \\
\mathrm{UV}-\mathrm{X}\end{array}$ & 19 & $\begin{array}{l}18 \mathrm{~mW} / \mathrm{cm}^{2} \text { for } 5 \mathrm{mins} \\
\text { CCL-VARIO }\end{array}$ & 12 & $\begin{array}{l}\text { Comparable visual acuity and } \\
\text { refractive outcomes. More } \\
\text { topographic flattening in the } \\
\text { conventional group compared to } \\
\text { accelerated group }\end{array}$ \\
\hline \multirow[t]{2}{*}{$\begin{array}{l}\text { Shetty et al. } \\
2015^{11}\end{array}$} & $\begin{array}{l}\text { Prospective } \\
\text { randomized } \\
\text { interventional study }\end{array}$ & 36 & $\begin{array}{l}3 \mathrm{~mW} / \mathrm{cm}^{2} \\
\text { for } 30 \mathrm{mins} \\
\text { Avedro } \mathrm{KXL}\end{array}$ & 36 & $9 \mathrm{~mW} / \mathrm{cm}^{2}$ for $10 \mathrm{mins}$ & 12 & \multirow{2}{*}{$\begin{array}{l}\text { Conventional group and } \\
\text { accelerated groups with } \\
\text { irradiance of } 9 \mathrm{~mW} / \mathrm{cm} 2 \text { and } \\
18 \mathrm{~mW} / \mathrm{cm}^{2} \text { showed better visual, } \\
\text { refractive and tomographic }\end{array}$} \\
\hline & & & & 33 & $18 \mathrm{~mW} / \mathrm{cm}^{2}$ for $5 \mathrm{mins}$ & & \\
\hline $\begin{array}{l}\text { Cummings } \\
\text { et al } 2016^{12}\end{array}$ & $\begin{array}{l}\text { Retrospective } \\
\text { interventional study }\end{array}$ & 66 & $\begin{array}{l}3 \mathrm{~mW} / \mathrm{cm}^{2} \\
\text { for } 30 \mathrm{mins}\end{array}$ & 36 & $9 \mathrm{~mW} / \mathrm{cm}^{2}$ for $10 \mathrm{mins}$ & 12 & $\begin{array}{l}\text { Accelerated corneal CXL is } \\
\text { effective in stabilizing } \\
\text { topographic parameters better } \\
\text { after } 12 \text { months } 9 \mathrm{~mW} / \mathrm{cm}^{2}\end{array}$ \\
\hline $\begin{array}{l}\text { Yildrim et al } \\
2017^{13}\end{array}$ & $\begin{array}{l}\text { prospective } \\
\text { comparative study }\end{array}$ & 74 & $\begin{array}{l}18 \mathrm{~mW} / \mathrm{cm}^{2} \\
\text { for } 5 \mathrm{mins}\end{array}$ & 72 & $30 \mathrm{~mW} / \mathrm{cm}^{2}$ for $3 \mathrm{mins}$ & 12 & $\begin{array}{l}\text { Comparable visual acuity, } \\
\text { refractive, keratometric }\end{array}$ \\
\hline
\end{tabular}

$\mathrm{UCVA}=$ Uncorrected distance Visual acuity, CDVA = Corrected distance visual acuity, SE $=$ Spherical equivalent, Ast $=$ Refractive astigmatism, Steep K = Steep keratometry, Sim K = Simulated keratometry, Kmax = Maximum or Apex Keratometry, CCT = Central corneal thickness

group during follow-up. One eye (4\%) in each group exhibited continued ectatic progression in terms of increase in Kmax of more than 1 dioptre.

\section{DISCUSSION}

The promising effects of AXL in halting disease progression by augmenting corneal strength and halving the surgical time with increased patient comfort have excited ophthalmologic researchers. Modified higher UVA irradiation intensity protocols with different time settings utilizing variable riboflavin solutions, soak time and CXL devices have been tested with the aim of achieving a short and equally effective procedure to date. ${ }^{3-13}$ In this particular study, both AXL showed equivalent improvement in UDVA, CDVA and topographic indices at 3, 6, 12 and 18 months follow-up except for flattening of keratometry $(\operatorname{sim} \mathrm{K}$, Steep K) that was significantly superior in AXL group 2 ( $p<0.007,0.023)$ at 18 months followup. Comparable clinical stabilization of disease (96\%) was achieved with optimum safety in each group. Failure rate of $4 \%$ in terms of continued progression, with no other adverse effects were documented in our study.

Significant improvements in our study in UDVA, CDVA along with reduction in SE and Ast at 6 and 12 months over baseline were consistent with refractive outcomes of prospective well designed interventional trials that recruited one arm of cohort to receive CXL irradiance protocol similar to our study. ${ }^{6,11,12}$ Hashemi et $\mathrm{al}^{8,9}$ and Al-Nawaeiseh et $\mathrm{al}^{14}$ in contrast to our results reported no significant improvements in refractive parameters at 12 months in patient receiving 5 minutes accelerated protocol. CXL alone without excimer laser is not considered a refractive procedure 
albeit, the effect on refractive parameters in different studies may be attributed to variable grades of keratoconus and associated difficult non repeatable subjective manifest refractions due to distorted multi focal optics of ectatic steep corneas. ${ }^{12}$

In present study, AXL groups demonstrated significant trend of flattening of keratometric indices $\mathrm{Kmax}$, steep $\mathrm{K}$, sim $\mathrm{K}$ within groups, with no superiority between the groups, except for sim $\mathrm{K}$ and steep $\mathrm{K}$ at 18 months follow-up. Although K max only just missed statistical significance at 18 months, a marked flattening of $2.7 \mathrm{D}$ was attained with 9 $\mathrm{mWatt} / \mathrm{cm}^{2}$ for 10 minutes regimen as compared to 1.5D in group 1. This trend of decrease in keratometric values was generally in accordance with results of previous studies of accelerated protocols. Shetty et $\mathrm{al}^{11}$ in a prospective interventional study on 138 keratoconic eyes, with randomization at radiance of 3 , 9, 18 or $30 \mathrm{mWatt} / \mathrm{cm}^{2}$ found that cross linking, flattening effect was abridged in higher irradiance and shorter duration treatments, in conformation with our results. However, the disease stability with AXL in each group in our study with no further corneal steeping at a long term follow up of 18 months is a significant clinical finding, even if it misses statistical significance between the groups.

The decrease in CCT is an indirect marker of efficacy of AXL, confirming compactness of collagen fibrils, apoptosis of stromal keratocytes and improved biomechanical stability. ${ }^{8,9,15}$ Hashemi et al $^{8,9}$ identified a statistically significant decrease in CCT at 18 months following AXL with $18 \mathrm{mWatt} / \mathrm{cm}^{2}$ for 5 minutes. We also observed a parallel decrease in CCT for both groups, without significance between the protocols at 18 months. Significant decrease in thinnest pachymetry was also reported for AXL $9 \mathrm{mWatt} / \mathrm{cm}^{2}$ in comparison against conventional $\mathrm{CXL}$ in literature. ${ }^{12,16,17}$

We lack the evidence of AXL induced structural change in anterior corneal stroma as anterior segment optical coherence tomography was not done in our study to assess the depth of demarcation line. In addition to this, the major limitation of this study was a relatively small sample size. We intend to follow our patients and publish our long term results to further validate efficacy of our procedures. We had $4 \%$ eyes in each group that experienced ectatic progression at 12 and 18 months, in terms of increase in Kmax, with no incidence of sterile infiltrates, persistent corneal haze and damage or loss of endothelial cells succumbing to corneal endothelial decompensation, akin to previous AXL trials. $12,18,19,20$

\section{CONCLUSION}

AXL protocols are safe and comparable in disease stability, and change in refractive parameters and topographic indices in progressive Keratoconus. These two modified high intensity protocols may be recommended alternatively in treatment of progressive keratoconus, considered fit for epithelium off CXL.

\section{ACKNOWLEDGEMENTS}

Mr Kashif Siddique, King Salman armed forces hospital, KSA Academic affairs (Research Unit) for helping in data analysis. Miss Paree Chera, Ex Optometrist, Armed forces institute of ophthalmology for helping in collection of data.

\section{Ethical Approval}

The study was approved by the Institutional review board/Ethical review board.

\section{Conflict of Interest}

Authors declared no conflict of interest

\section{REFERENCES}

1. Bunsen RW, Roscoe HE. Photochemical researchesPart V. On the measurement of the chemical action of direct and diffuse sunlight. Proc R Soc Lond. 1862; 12: 306-312.

http://rspl.royalsocietypublishing.org/content/12/306.

2. Wollensak G, Spoerl E, Seiler T. Riboflavin/ultraviolet-A- induced collagen cross linking for the treatment of keratoconus. Am J Ophthalmol. 2003; 135 (5): 620-627. https//doi.org/10.1016/S0002-9394(02)02220-1.

3. Krueger RR, Herekar S, Spoerl E. First proposed efficacy study of high versus standard irradiance and fractionated riboflavin/ultraviolet a cross-linking with equivalent energy exposure. Eye \& Contact Lens, 2014; 40 (6): 353-357. Doi: 10.1097/ICL.0000000000000095.

4. Schumacher S, Oeftiger L, Mrochen M. Equivalence of biomechanical changes induced by rapid and standard corneal cross linking, using riboflavin and ultraviolet radiation. Invest. Ophthalmol. Vis. Sci., 2011; 52 (12): 9048-9052.

Doi:10.1167/iovs.11-7818. 
5. Raiskup-Wolf F, Hoyer A, Spoerl E, Pillunat LE. Collagen cross linking with riboflavin and ultraviolet-A light in keratoconus: long-term results. J Cataract Refract Surg. 2008; 34 (5): 796-801. https://doi.org/10.1016/j.jcrs.2007.12.039.

6. O'Brart DPS. Corneal collagen cross-linking: a review. J Optom. 2014; 7 (3): 113-124. Doi: 10.1016/j.optom.2013.12.001.

7. Cınar Y, Cingü AK, Türkcü FM, Cınar T, Yüksel H, Ozkurt ZG, et al. Comparison of accelerated and conventional corneal collagen cross-linking for progressive keratoconus. Cutan Ocul Toxicol. 2014; 33 (3): 218-222. Doi: 10.3109/15569527.2013.834497.

8. Hashemi H, Fotouhi A, Miraftab M, Bahrmandy H, Seyedian MA, Amanzadeh K, et al. Short-term comparison of accelerated and standard methods of corneal collagen crosslinking. J Cataract Refract Surg. 2015; 41 (3): 533-540. Doi:10.1016/j.jcrs.2014.07.030

9. Hashemi H, Miraftab M, Seyedian MA, Hafezi F, Bahrmandy H, Heidarian S, et al. Long-term results of an accelerated corneal cross-linking protocol (18 $\mathrm{mW} / \mathrm{cm}^{2}$ ) for the treatment of progressive keratoconus. Am J Ophthalmol. 2015; 160 (6): 1164-1170. Doi: 10.1016/j.ajo.2015.08.027.

10. Chow VW, Chan TC, Yu M, Wong VW, Jhanji V. One-year outcomes of conventional and accelerated collagen cross linking in progressive keratoconus. Sci Rep. 2015; 5: 14425. Doi: 10.1038/srep14425.

11. Shetty R, Pahuja NK, Nuijts RM, Ajani A, Jayadev C, Sharma C, et al. Current protocols of corneal collagen cross-linking: Visual, refractive, and tomographic outcomes. Am J Ophthalmol. 2015; 160 (2): 243-249. Doi: 10.1016/j.ajo.2015.05.019.

12. Cummings AB, McQuaid R, Naughton S, Brennan E, Mrochen M. Optimizing corneal cross linking in the treatment of keratoconus: a comparison of outcomes after standard- and high-intensity protocols. Cornea, 2016; 35 (6): 814-822.

Doi:10.1097/ICO.0000000000000823.

13. Yıldırım Y, Olcucu O, Gunaydin ZK, Aqca A, Ozgurhan EB, Alagoz C, et al. Comparison of accelerated corneal collagen cross-linking types for treating keratoconus. Current eye research, 2017; 42 (7): 971-975. Doi:10.1080/02713683.2017.1284241.

14. Alnawaiseh M, Rosentreter A, Böhm MR, Eveslage M, Eter N, Zumhagen L. Accelerated $\left(18 \mathrm{~mW} / \mathrm{cm}^{2}\right)$ corneal collagen cross linking for progressive keratoconus. Cornea, 2015; 34 (11): 1427-1431.

Doi: 10.1097/ICO.0000000000000578.
15. Woo JH, Iyer JV, Lim L, Hla MH, Mehta JS, Chan CM, et al. Conventional versus accelerated collagen cross-linking for keratoconus: a comparison of visual, refractive, topographic and biomechanical outcomes. The Open Ophthalmology Journal, 2017; 11: 262. Doi:10.2174/1874364101711010262.

16. Akbar B, Intisar-ul-Haq R, Ishaq M, Fawad A, Arzoo S, Siddique K. Comparison of transepithelial corneal cross linking with epithelium-off crosslinking (epithelium-off CXL) in adult Pakistani population with progressive keratoconus. Taiwan J Ophthalmol, 2017; 7 (4):185. Doi: [10.4103/tjo.tjo_38_17].

17. Haq I, Fawad A, Saeed M, Humayun S, Islam Q, Arzoo S, et al. Comparison of rapid and conventional corneal collagen cross linking in patients having keratoconus. Pakistan Armed Forces Medical Journal, 2015; 1 (1): 105-109.

18. Koller T, Mrochen M, Seiler T. Complication and failure rates after corneal cross linking. J Cataract Refract Surg. 2009; 35 (8): 1358-1362. Doi:10.1016/j.jcrs.2009.03.035.

19. Cerman E, Toker E, Ozarslan Ozcan D. Transepithelial versus epithelium off cross linking in adults with progressive Keratoconus. J cataract Refract Surg. 2015; 41 (7): 1416-1425.

20. Lesniak SP, Hersh PS. Transepithelial corneal collagen cross linking for Keratoconus: six months results. J Cataract Refract Surg. 2014 Dec; 40 (12): 1971-1979.

\section{Authors' Designation and Contribution}

Bushra Akbar; Registrar: Study design, analysis of data, manuscript drafting and critical revision of final draft.

Imran Basit; Assistant Professor: Study design, analysis of data, manuscript drafting and critical revision of final draft.

Amjad Akram; Research Advisor: Critical revision of manuscript, final approval of draft for publication.

Maham Zahid; Research Associate: Data collection, analysis of data, interpretation of data, manuscript drafting and final approval of draft. 